\title{
Anneau vaginal à progestérone: aussi sûr pour maman que pour bébé
}

Population Council

Follow this and additional works at: https://knowledgecommons.popcouncil.org/departments_sbsr-rh

Part of the Demography, Population, and Ecology Commons, Family, Life Course, and Society Commons, International Public Health Commons, Maternal and Child Health Commons, and the Women's Health Commons How does access to this work benefit you? Let us know!

\section{Recommended Citation}

"Anneau vaginal à progestérone: aussi sûr pour maman que pour bébé," fact sheet. New York: Population Council, 2015. 


\section{ANNEAU VAGINAL À PROGESTÉRONE AUSSI SÛR POUR MAMAN QUE POUR BÉBÉ}

\section{L'anneau vaginal à progestérone (AVP) est un anneau vaginal contraceptif contenant de la progestérone. II a été prouvé qu'il s'agit d'une méthode contraceptive sûre et efficace pour les femmes qui allaitent.}

L'AVP est sans danger pour les mamans comme pour leurs bébés car il repose sur l'allaitement maternel et n'a pas d'effet sur la santé. Son effet contraceptif fonctionne en:

- renforçant les effets inhibiteurs de l'allaitement maternel sur l'ovulation;

- étendant l'aménorrhée post-partum.

\section{L'AVP N'AFFECTE PAS L'ALLAITEMENT}

Une mère qui allaite pourra le faire sans interruption. Elle pourra allaiter autant et aussi longtemps qu'elle l'aurait fait sans I'AVP. L'hormone naturelle de progestérone présente dans l'anneau n'affecte ni le volume de lait maternel produit par une femme, ni la fréquence des tétées. Au contraire, l'efficacité de la méthode repose sur un allaitement continu avec un minimum de 4 tétées par jour.

Un test clinique comparant les AVP et les DIU (dispositifs intra-utérins) prouve que les femmes utilisant des APG allaitaient aussi ou plus longtemps que les femmes sous IUD. Les motifs de sevrages étaient les mêmes pour les deux groups. (Sivin et al. 1997)

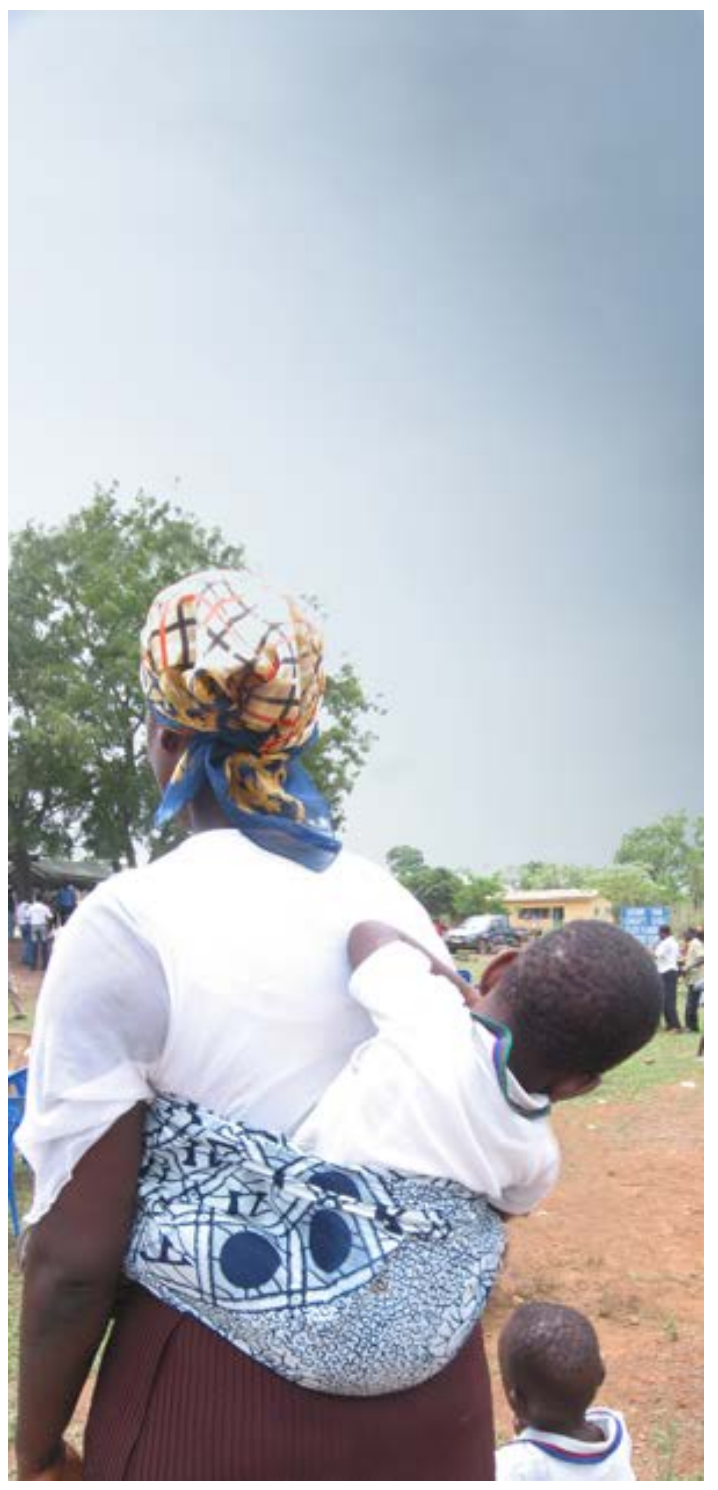




\section{EFFETS SUR LA SANTÉ DE L'ENFANT}

Il a été démontré que I'AVP n'avait aucun effet sur la croissance ou le développement des bébés dont les mères utilisent un anneau vaginal.

Des tests cliniques ont prouvé que les bébés dont les mères utilisaient un AVP ne présentaient aucune différence de croissance ou de développement par rapport à ceux dont les mères utilisaient un DIU au cuivre. (Nath and Sitruk-Ware 2010)

- Le poids des nourrissons dont les mères utilisaient un AVP ne différaient en rien du poids des bébés dont les mères utilisaient un DIU pendant la première année de vie. D'autres études cliniques ont reporté une croissance similaire du poids des bébés pendant la première année de vie. (Massai et al. 2005)

- La dose d'hormone délivrée par l'anneau est inférieure à celle normalement secrétée dans le sang par une femme en pleine ovulation. Ainsi, le nourrisson est exposé à une dose d'hormone bien inférieure à celle à laquelle il aurait normalement été exposé si sa mère avait retrouvé un cycle menstruel régulier.

- Avant une mère sous contraception, un bébé reçoit habituellement par le lait maternel de très faibles quantité de progestérone. Des estimation indiquent, par exemple, que les bébés de mères utilisant des implants reçoivent environ $5 \mathrm{mcg} / \mathrm{j}$ de progestérone, ce qui est très inférieur à la prise quotidienne maximale de $150 \mathrm{mcg} / \mathrm{j}$ préconisée par l'Agence Européenne des Médicaments (AEM).

- Moins de $10 \%$ de progestérone est biodisponible aux bébés, à cause de la faible teneur du lait maternel en progestérone et son élimination rapide après avoir traversé le tube digestif des nourrissons. (Nath and Sitruk-Ware 2010 : Massai et al. 2000)

\section{EFFETS SUR LES PARTENAIRES SEXUELS}

Les partenaires sexuels sont faiblement exposés à la progestérone contenue dans l'anneau et ne sont donc pas affectés par celle-ci. Tout au plus, certains hommes peuvent percevoir la présence de l'anneau. Des utilisateurs ont rapporté que l'anneau n'avait aucun effet sur leur plaisir ou celui de leur partenaire. (Nath and Sitruk-Ware 2010)

\section{MULTIPLIER LES OPTIONS CONTRACEPTIVES}

En 2015, I'AVP a été ajouté à la ligne directrice de I'utilisation de contraceptifs de la Liste des Médicaments Essentiels (LME) et Critères d'Éligibilité Médicale (CEM) de I'Organisation Mondiale de la Santé (OMS) destinée aux fournisseurs.

\section{SOMMAIRE}

L'AVP est un contraceptif sûr et efficace pour les mamans, leurs bébés et leurs partenaires sexuels. Cette méthode étend largement le gamme de contraceptifs à la disposition des femmes qui allaitent.

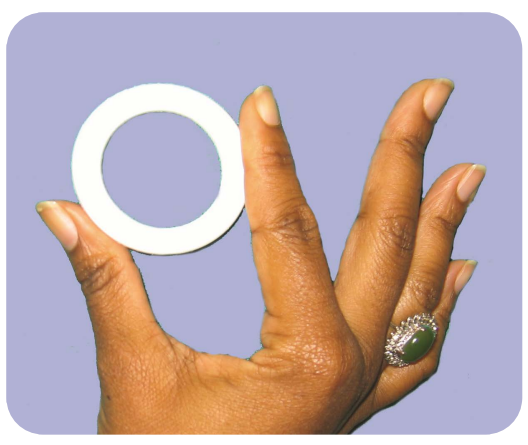

\section{Références}

Massai, Rebeca, Soledad Diaz, Ted Jackanicz, and Horacio B. Croxatto. 2000. "Vaginal rings for contraception in lactating women" Steroids 65: 703-707.

Massai, Rebeca, Eliana Quinteros, María Verónica Reyes, Roxana Caviedes, Ana Zepeda, Juan Carlos Montero and Horacio B. Croxatto. 2005.

"Extended use of a progesterone-releasing vaginal ring in nursing women: a phase II clinical trial" Contraception 72: 352-357.

Nath, Anita and Regine Sitruk-Ware. 2010. “Progesterone vaginal ring for contraceptive use during lactation" Contraception 82: 428-434.

Sivin, Irving, Soledad Díaz, Horacio B. Croxatto, et al. 1997.

"Contraceptives for lactating women: A comparative trial of a progesteronereleasing vaginal ring and the Copper T 380A IUD" Contraception 55: 225232.

Truitt, S.T., A.B. Fraser, Maria F. Gallo, L.M. Lopez, D.A. Grimes, and K.F. Schulz. 2003. "Combined hormonal versus nonhormonal versus progestinonly contraception in lactation" Cochrane Database of Systematic Reviews, Issue 2, Art. No.: CD003988. DOI: 10.1002/14651858.CD003988.

\section{POUR PLUS D'INFORMATIONS}

Pour plus d'informations concernant le Conseil, veuillez consulter notre site web www.popcouncil.org 